\title{
Amberlyst 15 Catalyzed Prenylation of Phenols: One-Step Synthesis of Benzopyrans
}

\author{
G. P. Kalena, A. Jain and A. Banerji*
}

Bio-Organic Division, Bhabha Atomic Research Centre, Mumbai-400085, India. Tel. 91-22-5563060; Fax: 91-22-5560750; E-mail: abanerji@magnum.barc.ernet.in

Received: 23 May 1997 / Accepted: 4 June 1997 / Published: 30 July 1997

\begin{abstract}
A convenient one-step synthesis of 2,2-dimethylchromans and chromenes using Amberlyst 15 as a catalyst is described.
\end{abstract}

Keywords: Amberlyst 15, 2,2-dimethylbenzopyrans, nuclear isoprenylation, eupatoriochromene, calanolides.

\section{Introduction}

The 2,2-dimethylbenzopyran system is frequently encountered in many natural products, some of which exhibit significant bioactivities [1]. This system is also present in the recently discovered the HIV inhibitory class of benzotripyrans [2]. Several approaches for the construction of 2,2-dimethylbenzopyrans have been reported [3]. Among them $\mathrm{H}_{3} \mathrm{PO}_{4}$ has been widely used in the nuclear isoprenylation of phenols $\mathbf{1}$ with isoprene (2) leading to the synthesis of 2,2-dimethylchromans 4 [4,5]. The obvious advantages of the solid catalysts in organic synthesis prompted us to explore the use of the macroreticular sulfonic acid cation exchange resin, Amberlyst $^{\circledR} 15$ for this condensation. We have found that Amberlyst 15 efficiently catalyses the condensation of phenols 1 with isoprene (2) in improved yields and better positional selectivity in the formation of 2,2dimethylchromans 4 . The method is further extended to the one-step synthesis of 2,2-dimethylchromenes 5 using 3hydroxy-3-methylbut-1-yne (3) as chromenylating agent (Scheme 1).

\section{Results and Discussion}

\section{Synthesis of 2,2-dimethylchromans 4}

For the synthesis of 2,2-dimethylchromans, the condensation of phenols 1 with isoprene (2) was optimized

* To whom correspondance should be addressed after extensive experimentation. Best result was obtained by the slow addition of $\mathbf{2}$ (1.1 equivalent in heptane) to the mixture of Amberlyst 15 and phenols 1 in THF at 65$70{ }^{\circ} \mathrm{C}$. After completion of the reaction, the desired products were isolated by the filtration of the resin catalyst and evaporation of the solvent from the filtrate followed by column chromatography. The products were characterised by comparing their physical and spectral data with the reported values. The recovered resin catalyst could be reused (at least four times). This procedure has better positional selectivity in the chroman formation. For example, condensation of hydroquinone (1a) with isoprene gave the mono prenylated product, 2,2-dimethyl-6hydroxychroman (4a) in $61 \%$ yield. Condensation using $\mathrm{H}_{3} \mathrm{PO}_{4}$ as described by Ahluwalia et al. [4] gave 4a in $13 \%$ yield along with mixture of two diprenylated products in

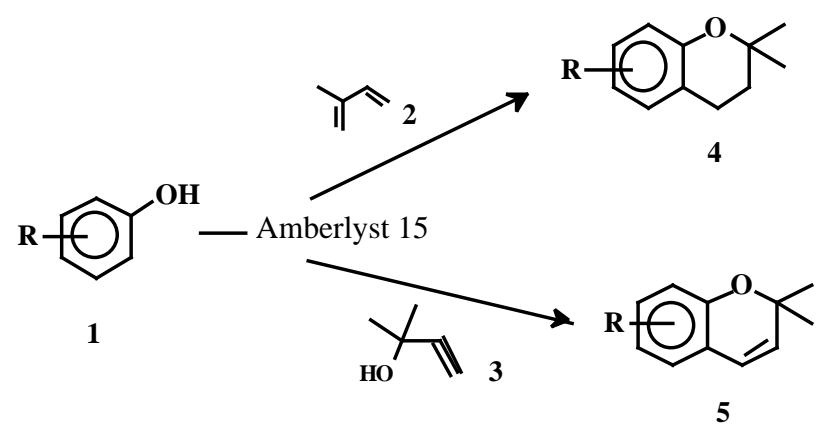

Scheme 1. 
equal proportions.

Earlier, we have shown that the compounds with $o$-hydroxy acetyl group can be conveniently transformed into variety of mixed polyheterocycles [3g]. Therefore, reactions were carried out with various acyl phenols. Thus, the reaction with resacetophenone (1e) gave two products, viz. 2,2-dimethyl-6-acetyl-7-hydroxychroman (4e, 31\%) and 2,2-dimethyl-6-acetyl-5-hydroxychroman (4f, 24\%) along with the recovery of $\mathbf{1 e}(32 \%)$. In contrast, lower yields (4f, 15\% and $\mathbf{4 g}, 17 \%$ ) are reported [5] with the use of $\mathrm{H}_{3} \mathrm{PO}_{4}$ as catalyst. Prenylation of phloroacetophenone (1g) with isoprene by the present method gave a mixture of two mono prenylated products, viz. 2,2-dimethyl-6-acetyl5,7-dihydroxychroman $(\mathbf{4 g}, 22 \%)$ and 2,2-dimethyl-8acetyl-5,7-dihydroxychroman (4h, 28\%) (useful intermediates for natural products synthesis [6]). Using $\mathrm{H}_{3} \mathrm{PO}_{4}$ as catalyst, formation of $\mathbf{4 h}$ is accompanied with substantial amount of the diprenylated products [7].

Chart 1. Prenylation of phenolics with isoprene using Amberlyst 15.

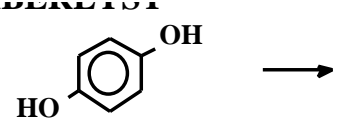

1a<smiles>CC1(C)CCc2cc(O)ccc2O1</smiles>
(68\%)

$4 a$<smiles>Oc1cccc(O)c1</smiles>

$1 b$<smiles>Oc1cc(O)cc(O)c1</smiles>

$1 d$<smiles>CCC(=O)c1ccc(O)cc1O</smiles>

$1 e$<smiles>CC(=O)c1c(O)cc(O)cc1O</smiles>

$1 g$<smiles>CC(=O)c1c(O)cccc1O</smiles>

$\mathbf{1 i}$<smiles>CCC(=O)c1c(O)cc(O)cc1O</smiles>

1j<smiles>CC1(C)CCc2ccc(O)cc2O1</smiles>

$4 b$ 4c

4d<smiles>CC(=O)c1cc2c(cc1O)OC(C)(C)CC2[13C](C)=O</smiles>

(31\%)

(24\%)

$4 \mathrm{e}$<smiles>CC(=O)c1c(O)cc2c(c1O)CCC(C)(C)O2</smiles>

(22\%)

$4 \mathrm{~g}$<smiles>CC(=O)c1c(O)cc(O)c2c1OC(C)(C)CC2</smiles>

(28\%)

$4 h$

(69\%)<smiles>CC(=O)c1c(O)ccc2c1OC(C)(C)CC2</smiles>

$4 \mathbf{i}$<smiles>CCC(=O)c1c(O)cc2c(c1O)CCC(C)(C)O2</smiles>

(26\%)

$4 \mathbf{j}$<smiles>CCC(=O)c1c(O)cc(O)c2c1OC(C)(C)CC2</smiles>

(26\%) 
<smiles>Oc1ccc(O)cc1</smiles>

$1 \mathbf{a}$<smiles>C#CC(C)(O)CCCCCCC</smiles>

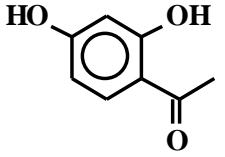

1e

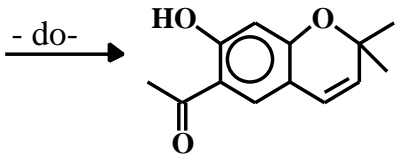

$5 \mathbf{e}$<smiles>CC(=O)c1ccc2c(c1O)C=CC(C)(C)O2</smiles>

$\mathbf{5 f}$

Scheme 2.

Similarly prenylation of phloropropiophenone (1j) with isoprene gave a mixture of two products, viz. 2,2-dimethyl6-propionyl-5,7-dihydroxychroman $(\mathbf{4 j}, 26 \%)$ and 2,2dimethyl-8-propionyl-5,7-dihydroxychroman (4k, 26\%). These compounds can serve as synthons for the nonnucleoside anti-HIV coumarin derivatives, calanolides [2], isolated from Calophyllum sp. The prenylation of 2,6dihydroxy acetophenone (1i) with isoprene gave $4 \mathbf{i}$ in $69 \%$ yield. The products obtained by prenylation of various phenols using the above method is given in Chart 1 .

\section{Synthesis of 2,2-dimethylchromenes 5}

Syntheses of 2,2-dimethylchromenes by the acid catalyzed reaction of phenols with the 3-hydroxy-3methylbut-1-yne (3) has been reported. However, yields are generally very poor $(<10 \%)[5,8]$. The above protocol was, therefore, extended to chromenylation. Thus, when reaction was carried out with 3 (in place of isoprene), 2,2dimethylchromenes could be obtained. Hence, condensation of $\mathbf{1}$ with $\mathbf{3}$ was carried out using Amberlyst 15 in refluxing benzene with azeotropic removal of water in a Dean-Stark apparatus. It is important that the reaction is monitored carefully as the undesired side products start appearing on prolonging the reaction time. The reaction products could be conveniently isolated and characterised as described above.
This strategy has been successfully used in the synthesis of some naturally occurring bioactive chromenes. (Scheme 2). 2,2-Dimethyl-6-hydroxychromene (5a) has been characterised as the anti-tumour principle of the plant, Agertina aromatica. 5a has now been synthesised in onestep in $25 \%$ yield by the condensation of $\mathbf{1 a}$ with $\mathbf{3}$. The earlier syntheses [9] of 5a involved several steps with lower yields. Similarly, condensation of $\mathbf{3}$ with resacetophenone (1e) gave a mixture of chromatographically separable two compounds, 2,2dimethyl-6-acetyl-7-hydroxychromene (5e, eupatoriochromene, 18\%) and 2,2-dimethyl-6-acetyl-5hydroxychromene (5f, 12\%) in $30 \%$ overall yield. Eupatoriochromene (5e) is the insecticidal principle of the plant, Eupatorium riparium Regel. Compared to reported methods, this is improved and shorter route to these chromenes [10-12].

\section{Mechanism of chromene formation}

The chroman formation by acid catalysis has already been discussed earlier [4]. The mechanism for the chromenylation, using macroreticular sulfonic acid cation exchange resin, might involve protonation of $\mathbf{3}$ followed by the elimination of water. The polymeric conjugate base may facilitate [13] the arene-anion attack giving the allenic intermediate which on electrocyclization [8] could result in

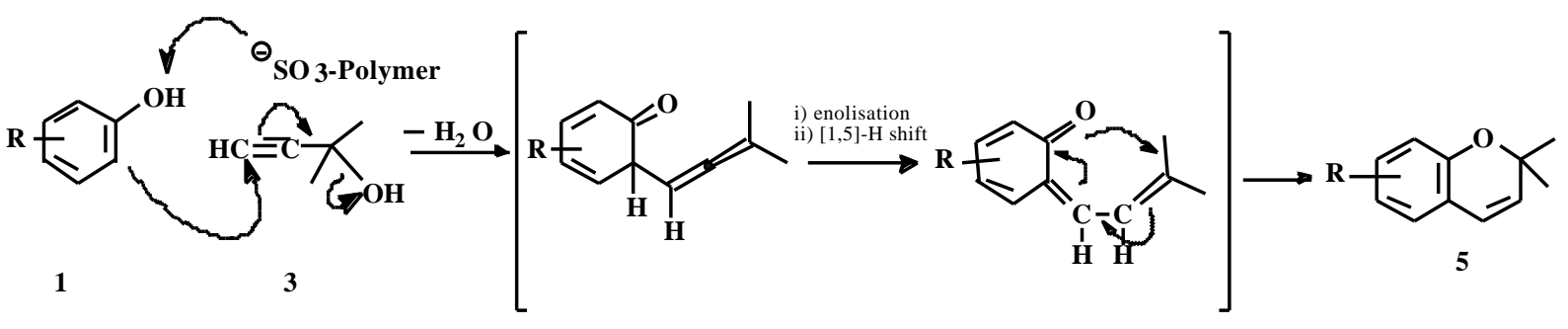

Scheme 3. 
the formation of 2,2-dimethylchromene (Scheme 3).

In summary, the use of macroreticular cation exchange resin Amberlyst 15 provides operationally simplified and improved method for the preparation of chromans and chromenes. In this one-step procedure, the products were isolated by simple filtration without the messy work up. In addition, like most of the solid supported reagents, the recovered resin retains its catalytic activity and can be recycled.

\section{Experimental Section}

\section{General}

All mp's are uncorrected. The IR (KBr) spectra were scanned with a Perkin-Elmer spectrophotometer model 783 and only the pertinent values are mentioned. The PMR spectra were recorded on a Varian EM-60 spectrometer (60 $\mathrm{MHz}$ ). The UV spectra were recorded in $\mathrm{MeOH}$ using a Shimadzu Graphicord UV-Visible 240 spectrophotometer. The mass spectra $(70 \mathrm{eV})$ were obtained with a Shimadzu GCMS-QP1000A mass spectrometer. The organic extracts were dried over anhydrous $\mathrm{Na}_{2} \mathrm{SO}_{4}$. Amberlyst 15 (Aldrich chemicals) was used as obtained. Though, Amberlyst 15 was used all through the experiments, use of cation exchange resin, Tulsion ${ }^{\circledR}$ T-42 (MP) $\mathrm{H}^{+}$(Thermex Corporation, Pune, India), also yielded equally good results. All the compounds were purified by column or thin layer chromatography $\left(\mathrm{SiO}_{2}\right)$.

\section{Typical procedure for the preparation of 2,2-dimethylchromans:}

\section{2,2-Dimethyl-6-hydroxychroman (4a)}

To a stirred mixture of Amberlyst 15 (3.1 g) and 1a (1.55 g, $15.0 \mathrm{mmol})$ in $\mathrm{THF}(3.5 \mathrm{~mL})$, at $65-70{ }^{\circ} \mathrm{C}$, isoprene $(1.6 \mathrm{~mL}, 16.0 \mathrm{mmol})$ in heptane $(5 \mathrm{~mL})$ was added dropwise over a period of $2 \mathrm{~h}$. After stirring for additional $30 \mathrm{~min}$, ether $(25 \mathrm{~mL})$ was added, the catalyst filtered off and washed with hot acetone $(2 \times 25 \mathrm{ml})$. Removal of the solvents followed by column chromatography of the residue gave 2,2-dimethyl-6hydroxychroman (4a). Yield $1.62 \mathrm{~g}$ (61\%); m.p. $74^{\circ} \mathrm{C}$ (lit. [14] 74-75 $\left.{ }^{\circ} \mathrm{C}\right)$; IR: 3205, 1360, 1215, $1190 \mathrm{~cm}^{-1}$; UV: $298 \mathrm{~nm} ; \mathrm{MS}:(\mathrm{m} / \mathrm{z}) 178\left(\mathrm{M}^{+}\right)$; PMR $\left(\mathrm{CDCl}_{3}\right): \delta 1.27(\mathrm{~s}$, $\left.6 \mathrm{H}, 2 \times \mathrm{CH}_{3}\right), 1.72(\mathrm{t}, \mathrm{J}=7 \mathrm{~Hz}, 2 \mathrm{H}, 3-\mathrm{H}), 2.67$ (t, J = $7 \mathrm{~Hz}$, $2 \mathrm{H}, 4-\mathrm{H}$ ), 4.50 (br. s, 1H, ArOH), 6.60 (m, 3H, 6-H, 7-H and $8-\mathrm{H})$.

\section{2,2-Dimethyl-7-hydroxychroman (4b)}

Colourless solid, yield $38 \%$; m.p. $68{ }^{\circ} \mathrm{C}$ (hexane) (lit. [17] $\left.72-73{ }^{\circ} \mathrm{C}\right)$; IR: $3250 \mathrm{~cm}^{-1}$; MS (m/z): $178\left(\mathrm{M}^{+}\right)$; PMR $\left(\mathrm{CDCl}_{3}\right): \delta 1.2\left(\mathrm{~s}, 6 \mathrm{H}, 2 \times \mathrm{CH}_{3}\right), 1.69(\mathrm{t}, \mathrm{J}=7 \mathrm{~Hz}$, $2 \mathrm{H}, 3-\mathrm{H}), 2.60(\mathrm{t}, 2 \mathrm{H}, \mathrm{J}=7 \mathrm{~Hz}, 4-\mathrm{H}), 5.6(\mathrm{~s}, 1 \mathrm{H},-\mathrm{OH})$, $6.18(\mathrm{~d}, \mathrm{~J}=2 \mathrm{~Hz}, 1 \mathrm{H}, \mathrm{H}-8), 6.22(\mathrm{dd}, \mathrm{J}=2 \mathrm{~Hz}$ and $8 \mathrm{~Hz}$, $1 \mathrm{H}, \mathrm{H}-6), 6.7$ (d, J = $8 \mathrm{~Hz}, 1 \mathrm{H}, 5-\mathrm{H})$.

\section{2,2-Dimethyl-5-hydroxychroman (4c)}

Colourless solid, yield $32 \%$; m.p. $120{ }^{\circ} \mathrm{C}$ (hexane) (lit. [17] $\left.121{ }^{\circ} \mathrm{C}\right)$; IR: $3225 \mathrm{~cm}^{-1}$; MS (m/z): $178\left(\mathrm{M}^{+}\right)$; PMR $\left(\mathrm{CDCl}_{3}\right): \delta 1.32\left(\mathrm{~s}, 6 \mathrm{H}, 2 \times \mathrm{CH}_{3}\right), 1.8(\mathrm{t}, \mathrm{J}=7 \mathrm{~Hz}$, $2 \mathrm{H}, 3-\mathrm{H}), 2.65$ (t, 2H, J = $7 \mathrm{~Hz}, 4-\mathrm{H}), 5.0$ (br s, 1H, OH), $6.33(\mathrm{~m}, 2 \mathrm{H}, 6-\mathrm{H}$ and $8-\mathrm{H}), 6.9(\mathrm{t}, \mathrm{J}=8 \mathrm{~Hz}, 1 \mathrm{H}, 5-\mathrm{H})$.

\section{2,2-Dimethyl-5,7-dihydroxychroman (4d)}

Colourless solid, yield 50\%; m.p. $162^{\circ} \mathrm{C}$ (lit. [17] 163$\left.164^{\circ} \mathrm{C}\right)$; IR: $3245 \mathrm{~cm}^{-1}$; UV: $269.8 \mathrm{~nm}$; PMR $\left(\mathrm{CD}_{3} \mathrm{SOCD}_{3}\right): \delta 1.2\left(\mathrm{~s}, 6 \mathrm{H}, 2 \times \mathrm{CH}_{3}\right), 1.69(\mathrm{t}, \mathrm{J}=7 \mathrm{~Hz}, 2 \mathrm{H}$, 3-H), 2.50 (t, 2H, J = $7 \mathrm{~Hz}, 4-\mathrm{H}), 5.56$ (s, 1H, 6-H), 5.75 (s, $1 \mathrm{H}, 8-\mathrm{H}), 8.5$ and 8.8 (2s, $1 \mathrm{H}$ each, $\mathrm{ArOH})$.

\section{2,2-Dimethyl-6-acetyl-7-hydroxychroman (4e)}

Colourless solid, yield, 31\%; m.p. $118^{\circ} \mathrm{C}$ (hexane) (lit. [15]. 118-119 ${ }^{\circ} \mathrm{C}$ ); IR: $1645 \mathrm{~cm}^{-1}$; MS: (m/z) 220 $\left(\mathrm{M}^{+}\right)$; PMR $\left(\mathrm{CDCl}_{3}\right): \delta 1.34\left(\mathrm{~s}, 6 \mathrm{H}, 2 \times \mathrm{CH}_{3}\right), 1.8(\mathrm{t}, \mathrm{J}=7$ $\mathrm{Hz}, 2 \mathrm{H}, 3-\mathrm{H}$ ), 2.48 (s 3H, $\left.\mathrm{CH}_{3} \mathrm{CO}\right), 2.73$ (t, 2H, J = $7 \mathrm{~Hz}$, 4-H), 6.3 (s, 1H, 8-H), 7.33 (s, 1H, 5-H), 12.42 (s, 1H, chelated $\mathrm{OH})$.

\section{2,2-Dimethyl-6-acetyl-5-hydroxychroman (4f)}

Colourless solid, yield 24\%, m.p. $70{ }^{\circ} \mathrm{C}$ (hexane) (lit. [16] 72-73 $\left.{ }^{\circ} \mathrm{C}\right)$ IR: $1650 \mathrm{~cm}^{-1}$; MS: (m/z) $220\left(\mathrm{M}^{+}\right)$; $\operatorname{PMR}\left(\mathrm{CDCl}_{3}\right): \delta 1.31\left(\mathrm{~s}, 6 \mathrm{H}, 2 \times \mathrm{CH}_{3}\right), 1.76(\mathrm{t}, \mathrm{J}=7 \mathrm{~Hz}$, 2H, 3-H), 2.48 (s 3H, CH ${ }_{3} \mathrm{CO}$ ), 2.64 (t, 2H, J = 7 Hz, 4-H), $6.20(\mathrm{~d}, \mathrm{~J}=9 \mathrm{~Hz}, 1 \mathrm{H}, 8-\mathrm{H}), 7.44$ (d, J = $9 \mathrm{~Hz}, 1 \mathrm{H}, 7-\mathrm{H})$, 12.59 (s, 1H, chelated $\mathrm{OH})$.

\section{2,2-Dimethyl-6-acetyl-5,7-dihydroxychroman (4g)}

Pale yellow solid, yield 22\%; m.p. $230{ }^{\circ} \mathrm{C}$ (ether/hexane) (lit. [18] $229{ }^{\circ} \mathrm{C}$ ); IR: 1649, 1614, 1588, $1565 \mathrm{~cm}^{-1}$; MS: (m/z) $236\left(\mathrm{M}^{+}\right)$; PMR $\left(\mathrm{CD}_{3} \mathrm{COCD}_{3}\right): \delta$ $1.46\left(\mathrm{~s}, 6 \mathrm{H}, 2 \times \mathrm{CH}_{3}\right), 1.79(\mathrm{t}, \mathrm{J}=7 \mathrm{~Hz}, 2 \mathrm{H}, 3-\mathrm{H}), 2.66(\mathrm{~s}$, $\left.3 \mathrm{H}, \mathrm{CH}_{3} \mathrm{CO}\right), 2.6$ (t, 2H, J = $\left.7 \mathrm{~Hz}, 4-\mathrm{H}\right), 6.06$ (s, 1H, 8-H), 13.09 (s, 1H, chelated $\mathrm{OH})$.

\section{2,2-Dimethyl-8-acetyl-5,7-hydroxychroman (4h)}

Pale yellow solid, yield 28\%; m.p. $145-146{ }^{\circ} \mathrm{C}$ (benzene) (lit. [18] $150{ }^{\circ} \mathrm{C}$ ); IR: 1621, $1604 \mathrm{~cm}^{-1}$; UV: $291 \mathrm{~nm}$; MS: (m/z) $236\left(\mathrm{M}^{+}\right)$; PMR $\left(\mathrm{CD}_{3} \mathrm{COCD}_{3}\right): \delta 1.33$ (s, 6H, $2 \times \mathrm{CH}_{3}$ ), 1.79 (t, J = $\left.7 \mathrm{~Hz}, 2 \mathrm{H}, 3-\mathrm{H}\right), 2.63$ (s 3H, $\left.\mathrm{CH}_{3} \mathrm{CO}\right), 2.56(\mathrm{t}, 2 \mathrm{H}, \mathrm{J}=7 \mathrm{~Hz}, 4-\mathrm{H}), 5.92(\mathrm{~s}, 1 \mathrm{H}, 6-\mathrm{H})$, $9.56(\mathrm{~s}, 1 \mathrm{H}, \mathrm{ArOH}), 14.1$ (s, 1H, chelated $\mathrm{OH})$. 


\section{2,2-Dimethyl-8-acetyl-7-hydroxychroman (4i)}

Pale yellow solid, yield 69\%; m.p. 45-46 ${ }^{\circ} \mathrm{C}$ (ether/hexane) (lit. [19] 44-45 $\left.{ }^{\circ} \mathrm{C}\right)$; IR: $1630 \mathrm{~cm}^{-1}$; UV: $291.4 \mathrm{~nm}$; MS: (m/z) $220\left(\mathrm{M}^{+}\right)$; PMR $\left(\mathrm{CDCl}_{3}\right): \delta 1.4(\mathrm{~s}$, $6 \mathrm{H}, 2 \times \mathrm{CH}_{3}$ ), $1.76(\mathrm{t}, \mathrm{J}=7 \mathrm{~Hz}, 2 \mathrm{H}, 3-\mathrm{H}), 2.66$ (s $3 \mathrm{H}$, $\left.\mathrm{CH}_{3} \mathrm{CO}\right), 2.64(\mathrm{t}, 2 \mathrm{H}, \mathrm{J}=7 \mathrm{~Hz}, 4-\mathrm{H}), 6.5(\mathrm{~d}, \mathrm{~J}=9 \mathrm{~Hz}, 1 \mathrm{H}$, H-6), 7. 13 (d, J = 9 Hz, 1H, H-5), 12.59 (s, 1H, chelated $\mathrm{OH})$.

\section{2,2-Dimethyl-6-propionyl-5,7-dihydroxychroman (4j)}

Pale yellow solid, yield 26\%; m.p. $162{ }^{\circ} \mathrm{C}$ (ether/hexane); IR: 1635, 1588, $1560 \mathrm{~cm}^{-1}$; UV: $289.9 \mathrm{~nm}$; MS: $(\mathrm{m} / \mathrm{z}) 250\left(\mathrm{M}^{+}\right)$; PMR $\left(\mathrm{CDCl}_{3}\right): \delta 1.1(\mathrm{t}, \mathrm{J}=7 \mathrm{~Hz}, 3 \mathrm{H}$, $\left.\mathrm{CH}_{3} \mathrm{CH}_{2}\right), 1.33\left(\mathrm{~s}, 6 \mathrm{H}, 2 \times \mathrm{CH}_{3}\right), 1.76(\mathrm{t}, \mathrm{J}=6 \mathrm{~Hz}, 2 \mathrm{H}, 3-$ $\mathrm{H}), 2.6(\mathrm{t}, 2 \mathrm{H}, \mathrm{J}=6 \mathrm{~Hz}, 4-\mathrm{H}), 3.1(\mathrm{q}, \mathrm{J}=7 \mathrm{~Hz}, 3 \mathrm{H}$, $\left.\mathrm{CH}_{2} \mathrm{CO}\right), 5.7(\mathrm{~s}, 1 \mathrm{H}, \mathrm{OH}), 6.2(\mathrm{~s}, 1 \mathrm{H}, 8-\mathrm{H}), 13.5(\mathrm{~s}, 1 \mathrm{H}$, chelated $\mathrm{OH}$ ).

\section{2,2-Dimethyl-8-propionyl-5,7-hydroxychroman (4k)}

Pale yellow solid, yield 26\%; m.p. $133{ }^{\circ} \mathrm{C}$ (ether/hexane); IR: 1621, $1603 \mathrm{~cm}^{-1}$; UV: $290.5 \mathrm{~nm}$; MS: $(\mathrm{m} / \mathrm{z}) 250\left(\mathrm{M}^{+}\right)$; PMR $\left(\mathrm{CDCl}_{3}\right): \delta 1.16(\mathrm{t}, \mathrm{J}=7 \mathrm{~Hz}, 3 \mathrm{H}$, $\left.\mathrm{CH}_{3} \mathrm{CH}_{2}\right), 1.36\left(\mathrm{~s}, 6 \mathrm{H}, 2 \times \mathrm{CH}_{3}\right), 1.76(\mathrm{t}, \mathrm{J}=7 \mathrm{~Hz}, 2 \mathrm{H}, 3-$ $\mathrm{H}), 2.6(\mathrm{t}, 2 \mathrm{H}, \mathrm{J}=7 \mathrm{~Hz}, 4-\mathrm{H}), 3.03(\mathrm{q}, 2 \mathrm{H}, \mathrm{J}=7 \mathrm{~Hz}$, $\left.\mathrm{CH}_{2} \mathrm{CO}\right), 6.0(\mathrm{~s}, 1 \mathrm{H}, 6-\mathrm{H}), 14.1$ (s, 1H, chelated $\left.\mathrm{OH}\right)$.

\section{Typical procedure for the preparation of} 2,2-dimethylchromenes

\section{2,2-Dimethyl-6-hydroxychromene (5a)}

To a suspension of Amberlyst $15 \quad(4.4 \mathrm{~g})$ and hydroquinone (1a) $(1.1 \mathrm{~g}, 10.0 \mathrm{mmol})$ in benzene $(50 \mathrm{~mL})$, 3-hydroxy-3-methylbut-1-yne (3) $(12.0 \mathrm{mmol})$ in benzene $(50 \mathrm{ml})$ was added dropwise during $0.5 \mathrm{~h}$. The water generated in the reaction was continuously removed by a Dean-Stark apparatus and the reaction was monitored by TLC. After $45 \mathrm{~min}$, when the side products started to appear (TLC), the reaction was terminated and worked up as before. The residue was passed through a column of Florisil $(25.0 \mathrm{~g})$ and eluted with pet. ether. 5a was isolated as a colourless solid by preparative layer chromatography, yield 0.44 g (25\%); m.p. $86^{\circ} \mathrm{C}$ (lit. [10] 86-87 ${ }^{\circ} \mathrm{C}$ ) IR: $3500,1635,1600,1465 \mathrm{~cm}^{-1}$; UV: 260, $329 \mathrm{~nm}$; MS $(\mathrm{m} / \mathrm{z}): 176\left(\mathrm{M}^{+}\right)$; PMR $\left(\mathrm{CCl}_{4}\right): \delta 1.42\left(\mathrm{~s}, 6 \mathrm{H}, 2 \times \mathrm{CH}_{3}\right), 5.0$ (bs, 1H, OH), $5.56(\mathrm{~d}, \mathrm{~J}=9 \mathrm{~Hz}, 1 \mathrm{H}, 3-\mathrm{H}), 6.2(\mathrm{~d}, \mathrm{~J}=9 \mathrm{~Hz}$, $1 \mathrm{H}, 4-\mathrm{H}), 6.37-6.56$ (m, 3H, ArH).

\section{Reaction of 2,4-dihydroxyacetophenone (1e) with 3}

Compound $\mathbf{3}$ was reacted with $\mathbf{1 a}$ to give a mixture $\mathbf{5 e}$ and 5f, overall yield $30 \%$. The reaction products were separated by preparative TLC (pet. ether/EtOAc, 95:5).

\section{2,2-Dimethyl-6-acetyl-7-hydroxychromene (5e)}

Yield: $18 \%$; m.p. $75^{\circ} \mathrm{C}$ (lit. [17] $76^{\circ} \mathrm{C}$ ); IR: 2980, 2930, $1630 \mathrm{~cm}^{-1}$; UV: 232, $256 \mathrm{~nm}$; MS (m/z): $218\left(\mathrm{M}^{+}\right)$; PMR $\left(\mathrm{CCl}_{4}\right): \delta 1.44$ (s, 6H, $\left.2 \times \mathrm{CH}_{3}\right), 2.52$ (s 3H, $\mathrm{CH}_{3} \mathrm{CO}$ ), $5.53(\mathrm{~d}, \mathrm{~J}=9 \mathrm{~Hz}, 1 \mathrm{H}, \mathrm{CH}), 6.3(\mathrm{~s}, 1 \mathrm{H}, \mathrm{H}-8), 6.27$ (d, J = 9 $\mathrm{Hz}, 1 \mathrm{H}, \mathrm{CH}), 7.3$ (s, 1H, H-5), 12.59 (s, 1H, chelated $\mathrm{OH})$.

\section{2,2-Dimethyl-6-acetyl-5-hydroxychromene (5f)}

Yield: $12 \%$; Pale yellow solid, m.p. $104^{\circ} \mathrm{C}$ (pentane) (lit. [12] 104-105 ${ }^{\circ} \mathrm{C}$ ); IR: 1650, $1625 \mathrm{~cm}^{-1}$; UV: 265, 303 $\mathrm{nm} ; \mathrm{MS}(\mathrm{m} / \mathrm{z}): 218\left(\mathrm{M}^{+}\right)$; PMR $\left(\mathrm{CCl}_{4}\right): \delta 1.4(\mathrm{~s}, 6 \mathrm{H}, 2 \mathrm{x}$ $\left.\mathrm{CH}_{3}\right), 2.49$ (s, 3H, $\left.\mathrm{CH}_{3} \mathrm{CO}\right), 5.52(\mathrm{~d}, \mathrm{~J}=7 \mathrm{~Hz}, 1 \mathrm{H}, 3-\mathrm{H})$, $6.23(\mathrm{~d}, \mathrm{~J}=9 \mathrm{~Hz}, 1 \mathrm{H}, 4-\mathrm{H}), 6.72(\mathrm{~d}, \mathrm{~J}=9 \mathrm{~Hz}, 1 \mathrm{H}, 8-\mathrm{H})$, $7.79(\mathrm{~d}, \mathrm{~J}=9 \mathrm{~Hz}, 1 \mathrm{H}, 7-\mathrm{H}), 12.89$ (s, 1H, chelated $\mathrm{OH})$.

\section{References}

1. (a) Nunata, A.; Kanbara, S.; Takahashi, C.; Fujiki, R.; Yoneda, M.; Usami, Y.; Fujita, E. Phytochem. 1992, 31, 1209. (b) Brown, P. E.; Lewis, R.; Waring, M. A. J. Chem. Soc. Perkin Trans. I 1990, 2979. (c) Srivastava, R.; Proksch, P. Entomol. Gener. 1991, 15, 265. (d) Bergmann, R.; Gericke, R. J. Med. Chem. 1990, 33, 492. (e) Bergmann, R.; Harting, J.; Lues, L.; Schittenhelm, C.; Gericke, R. J. Med. Chem. 1991, 34, 3074. (f) Merrill, G. B.; J. Chem. Ecology 1989, 15, 2073. (g) Satoh, Y.; Stanton, J. L.; Hutchison, A. J.; Libby, A. J.; Kowalski, T. J.; Lee, W. H.; White, D. H.; Kimble, E. F. J. Med. Chem. 1993, 36, 3580.

2. (a) Kashman, Y.; Gustafson, K. R.; Fuller, R. W.; Cardellina II, J. H.; McMahon, J. B.; Currens, M. J.; Buckheit, Jr., R. W.; Hughes, S. H.; Cragg, G. M.; Boyd, M. R. J. Med. Chem. 1992, 35, 2735. (b) Currens, M. J.; Mariner, J. M.; McMahon, J. B.; Boyd, M. R.; J. Pharmacol. Exp. Ther. 1996, 279, 652. (c) Currens, M. J.; Gulakowski, R. J.; Mariner, J. M.; Moran, R. A.; Buckheit, R. W. Jr.; Gustafson, K. R.; McMahon, J. B.; Boyd, M. R. J. Pharmacol. Exp. Ther. 1996, 279, 645-651. (d) Hizi, A.; Tal, R.; Shaharabany, M.; Currens, M. J.; Boyd, M. R.; Hughes, S. H.; McMahon, J. B. Antimicrob. Agents Chemother. 1993, 37, 1037.

3. (a) Livingstone, R.; Watson, R. B. J.Chem. Soc. 1956, 3701. (b) Spath, E.; Hillel, R. Ber. Dtsch. Chem. Ges. 1939, 72, 963 and 2093. (c) Mukerjee, S.K.; Sarkar, S. C.; Seshadri, T.R. Tetrahedron 1969, 25, 1063. (d) Banerji, A.; Goomer, N. C.; Indian J. Chem. 1981, 20B, 144. (e) Ahluwalia, V. K. J. Chem. Soc., Perkin Trans. 1 1982, 335. (f) Bandaranayake, W. M.; Crombie, L.; Whiting, D. A. J. Chem. Soc. (C) 1970, 811. (g) Banerji, A.; Kalena, G. P.; Heterocycle 1989, 28, 711 and references therein. 
4. Ahluwalia, V. K.; Arora, K. K. J. Chem. Soc. Perkin Trans. 1 1982, 335.

5. Ahluwalia, V. K.; Arora, K. K. Tetrahedron 1981, 37, 1437.

6. Schweizer, E. E.; Meeder-Nycz, D. In Chromenes, Chromanones and Chromones; Ellis, G. P. Ed.; J. Wiley and Sons: New York, 1975, p. 55.

7. Ahluwalia, V. K.; Arora, K. K.; Jolly, R. S. Acta Chim. Hung. 1983, 114, 117.

8. Zsindely, J.; Schmid, H. Helv. Chim. Acta 1968, 51, 1510 .

9. (a) Howard, B. M.; Clarkson K.; Bernstein, R.L. Tetrahedron Lett. 1979, 4449. (b) Banerji, A.; Goomer, N. C. Indian J. Chem. 1984, 23B, 885.

10. Steelink, C.; Marshall, G. J. Org. Chem. 1979, 44, 1429.

11. Bohlman, F.; Buhmann, U. Chem. Ber. 1972, 105, 863.
12. Mukerjee, S. K.; Sarkar, S. C.; Seshadri, T. R. Indian J. Chem. 1970, 8, 861.

13. Tachibana, Y. Bull. Chem. Soc. Japan 1980, 555.

14. Miller, J. A.; Wood, H. C. S. J. Chem. Soc. C $\mathbf{9 9 6 8 ,}$ 1837.

15. Bajwa, B. S.; Lal P.; Seshadri, T. R. Indian J. Chem. 1973, 11, 100 .

16. Nickl, J. Chem. Ber. 1958, 91, 1372.

17. Bridge, W.; Croker, A. J.; Cubin T.; Robertson, A. J. Chem. Soc. 1937, 1530.

18. Backouse T.; Robertson, A. J. Chem. Soc. 1939, 1257.

19. Miranda, M. A.; Primo J.; Tormos, R. Tetrahedron 1989, 45, 7593.

20. Anthonsen, T. Acta Chem. Scand. 1969, 23, 3605.

Sample Availability: not available. 